\title{
SCIENTIFIC REPORTS

\section{OPEN Enhanced STAT3 phosphorylation and PD-L1 expression in myeloid dendritic cells indicate impaired IL-27Ralpha signaling in type 1 diabetes}

\author{
Z. Parackova ${ }^{1 *}$, P. Vrabcova ${ }^{1}$, I. Zentsova ${ }^{1}$, J. Kayserova ${ }^{1}$, I. Richtrova ${ }^{2}$, L. Sojka ${ }^{1,2}$, \\ K. Stechova ${ }^{3}$, Z. Sumnik ${ }^{4}$ \& A. Sediva ${ }^{1}$
}

Interleukin 27 (IL-27), a member of the IL-12 family, is important for T cell differentiation; however, little is known about its effect on dendritic cells (DCs). IL-27 can activate multiple signaling cascades, including the JAK/STAT pathway, and depending on the setting it can both promote and antagonize inflammatory responses. An anti-inflammatory function of IL-27 has been reported in several autoimmune diseases; however, in type 1 diabetes (T1D), an autoimmune disease where autoreactive cytotoxic T cells attack insulin-producing beta cells, IL-27 has been shown to have a dual role and contradictory effects. Here, we show impaired IL-27 signaling in a large cohort of T1D patients $(n=51)$ compared to age- and gender-matched healthy donors. Increased expression of the IL-27 receptor subunit IL-27Ralpha mRNA in purified myeloid DCs ( $m D C s)$, detected by gene expression microarrays was mirrored by enhanced signal transduction in T1D mDCs in response to IL-27 stimulation. Higher STAT phosphorylation in T1D patients was also accompanied by elevated expression of the inhibitory molecules $P D-L 1, P D-L 2$ and $P D-1$, which may suggest not only immunomodulatory mechanisms of IL-27 in T1D but also a compensatory effort of T1D dendritic cells against the ongoing inflammation.

Type 1 diabetes (T1D) is a type 1 helper T cell-mediated autoimmune disease caused by the destruction of beta cells in the Langerhans islets of the pancreas. However, the involvement in disease development and progression is not limited to autoreactive T cells; in fact, a wide variety of immune cell populations producing different cytokines accompany the inflammatory response (insulitis) within the islets. Cytokines play pivotal roles in immune system activation and have been extensively studied in autoimmune diseases, including T1D ${ }^{1-3}$.

Interleukin 27 (IL-27) is a heterodimeric member of the IL-12 family, is composed of two subunits, p28 and EBV-induced gene 3 (EBI3) and is produced primarily by activated antigen-presenting cells $s^{4,5}$. The role of IL-27 is enigmatic because it can induce both pro- and anti-inflammatory responses. Although the first animal studies described IL-27 as an initiator of the Th1 response ${ }^{5,6}$, it was later found that IL-27 has broad inhibitory effects on Th1, Th2, and Th17 subsets and can induce IL-10-producing regulatory T cells $(\operatorname{Tr} 1)$ in both mice and humans ${ }^{7-9}$.

IL-27 signals through a heterodimeric receptor composed of two subunits, gp130 and IL-27Ralpha (formerly called TCCR or WSX-1), which activate the STAT1 and STAT3 signaling pathways ${ }^{10}$. IL-27Ralpha exists in both a membrane and soluble form. Soluble IL-27Ralpha inhibits IL-27 binding to the cell surface and inhibits IL-27 signaling ${ }^{11}$.

IL-27 is known to act directly on T cells, suppressing their differentiation to effector T cells. Less is known about the effect of IL-27 on dendritic cells (DCs) ${ }^{9,12,13}$. IL-27 signaling in murine DCs limits pathogenic T cell

\footnotetext{
${ }^{1}$ Department of Immunology, 2nd Faculty of Medicine, Charles University and Motol University Hospital, Prague, Czech Republic. ${ }^{2}$ Sotio, A. S., Prague, Czech Republic. ${ }^{3}$ Department of Internal Medicine, 2nd Faculty of Medicine, Charles University and Motol University Hospital, Prague, Czech Republic. ${ }^{4}$ Department of Pediatrics, 2nd Faculty of Medicine, Charles University and Motol University Hospital, Prague, Czech Republic. *email: zuzana.parackova@ fnmotol.cz
} 


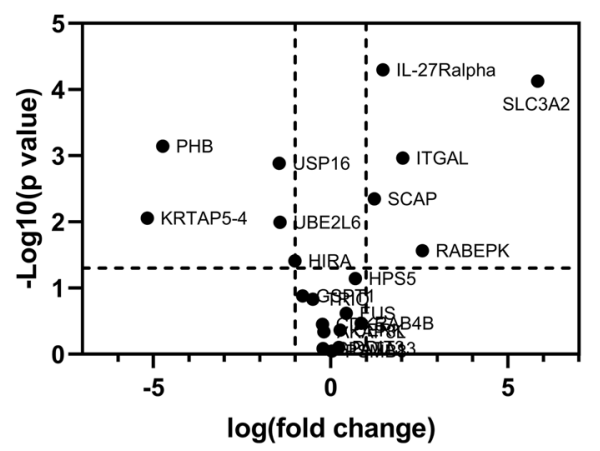

B

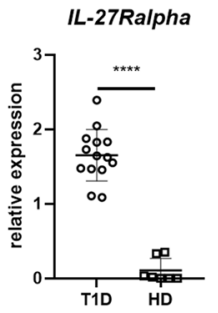

C

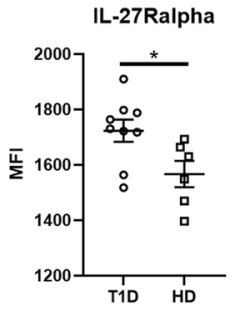

D

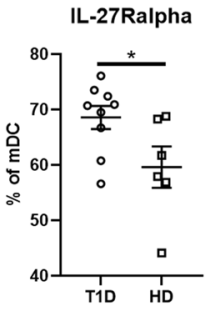

E

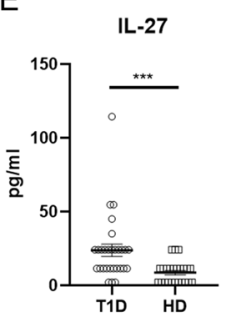

$\mathrm{F}$

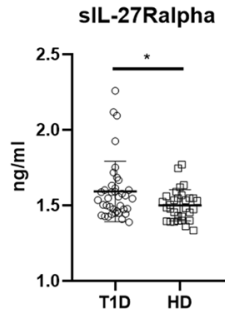

Figure 1. IL-27Ralpha expression. (A) Volcano plot of differentially expressed genes in T1D patients compared to healthy controls. Analysis of gene arrays was performed on sorted myeloid dendritic cells (mDCs) from T1D patients $(\mathrm{n}=20)$ and healthy controls $(\mathrm{n}=10)$. (B) IL-27Ralpha expression validation by RT PCR normalized to GADPH. Analysis was performed on sorted mDCs from 14 T1D patients and 7 healthy donors (HD). Surface expression of IL-27Ralpha on $\mathrm{mDC}$ expressed as (C) MFI and (D) percentage of IL-27Ralpha + CD11c + mDCs detected by flow cytometry in 9 T1D patients and 6 HD. (E) Serum levels of IL-27 in 30 T1D patients and 23 healthy donors detected by Luminex. (F) Serum levels of soluble IL-27Ralpha in T1D patients $(\mathrm{n}=40)$ and healthy donors $(\mathrm{n}=32)$ detected by ELISA. Statistical analysis was performed using the Mann-Whitney unpaired $t$-test. Values of $\mathrm{p}<0.05(*), \mathrm{p}<0.01(* *), \mathrm{p}<0.001(* * *)$ and $\mathrm{p}<0.0001(* * * *)$ were considered statistically significant.

responses, diminishes NLRP3 inflammasome activation and limits the ability of DCs to induce Th1 and Th17 differentiation and suppress autoimmune processes ${ }^{14}$.

The role of IL-27 in various autoimmune diseases has been reported ${ }^{15}$. IL-27 activates multiple signaling cascades and has both anti- and proinflammatory activities in different autoimmune diseases. However, with some exceptions, the anti-inflammatory effect of IL-27 has been mostly observed ${ }^{15}$.

In T1D, the role of IL-27 has been insufficiently investigated, and the studies are conflicting. Wang et al. showed that IL-27 has a pathogenic role in T cell-mediated autoimmune diabetes, using (nonobese diabetic) NOD mice ${ }^{16}$. On the other hand, Fujimoto et al. reported that mice lacking IL-27 or IL-27 receptor subunit had increased blood sugar levels, islet infiltration and proinflammatory cytokine levels in a streptozotocin-induced diabetes model ${ }^{17}$, suggesting that IL-27 could represent a potential target for therapeutic strategy. In humans, newly diagnosed T1D patients have increased IL-27 levels in the peripheral blood, and those levels strongly correlate with circulating Th17 cytokines, supporting the hypothesis that IL-27 can have a regulatory function ${ }^{3}$. An association between IL-27 polymorphisms and T1D was also reported in one genome-wide association study ${ }^{18}$, but this association was not confirmed in another report ${ }^{19}$.

In this study, we report for the first time the increased expression of IL-27Ralpha in myeloid dendritic cells (mDCs) in T1D patients, resulting in higher STAT3 phosphorylation and $P D-L 1$ expression in mDCs, suggesting a substantial immunomodulatory role in T1D pathogenesis.

\section{Results}

Increased IL-27Ralpha expression in myeloid dendritic cells in T1D patients. By performing gene array analysis, we discovered that the myeloid dendritic cells (mDCs) of T1D patients $(\mathrm{n}=20)$ expressed significantly more $I L-27$ Ralpha compared to healthy donors $(\mathrm{n}=10)$ (Fig. 1A). This finding was confirmed by real-time PCR analysis, whereby increased levels of $I L-27$ Ralpha were detected in purified patient mDCs compared to healthy donor mDCs (Fig. 1B). Flow cytometry verified the results on the protein level. Not only is the percentage of mDCs expressing IL-27Ralpha higher in T1D patients, but also the receptor subunit expression on the cells is elevated (Fig. 1C,D). No difference in the expression of the second subunit of IL-27 receptor, gp130, was found (Suppl. Fig. 1A). Notably, we have also examined the expression of metalloproteinase ADAM17, which regulates IL-27Ralpha subunit shedding from the cell surface and found lower expression on T1D mDC's surface (Suppl. Fig. 1B), thus elucidating the enhanced IL-27Ralpha expression on mDCs. Next, we established, that the higher 
A
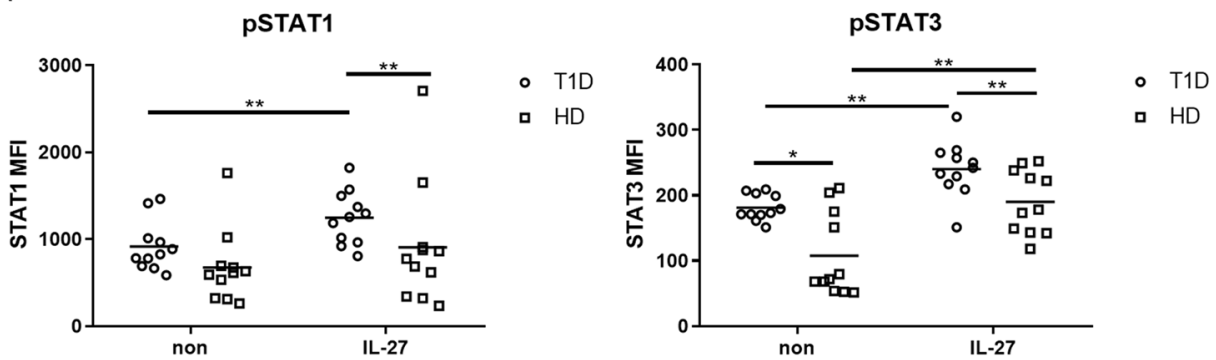

B
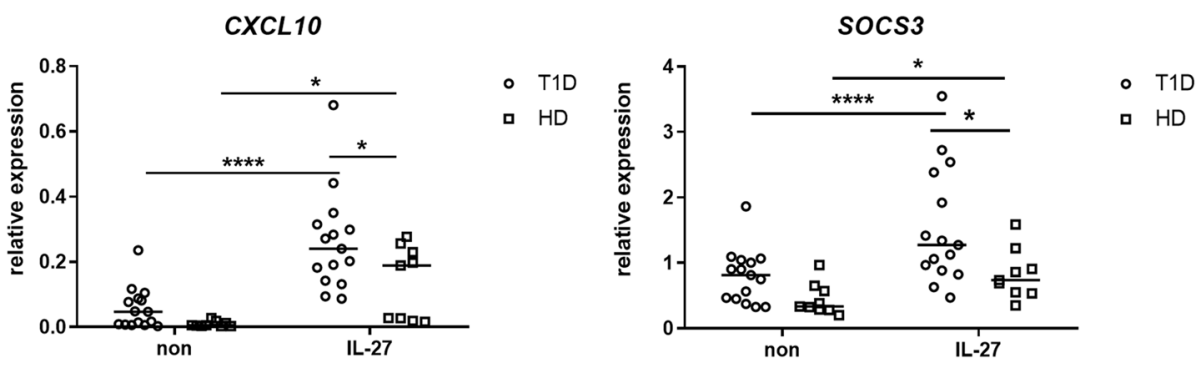

Figure 2. IL-27 signaling. (A) STAT1 (Tyr701) and STAT3 (Tyr705) phosphorylation in myeloid dendritic cells (mDCs) was analyzed by phosphoflow after whole blood from T1D patients $(\mathrm{n}=11)$, and healthy donors (HD, $\mathrm{n}=11$ ) was stimulated with $100 \mathrm{ng} / \mathrm{ml}$ rhIL-27 for 15 minutes. Values are expressed as MFI (mean fluorescence intensity). (B) Expression of STAT1-induced CXCL10 and STAT3-induced SOCS3 after rhIL-27 (100 ng/ $\mathrm{ml})$ exposure in T1D patients $(\mathrm{n}=16)$ and healthy donors PBMCs $(\mathrm{n}=9)$ was normalized to $G A D P H$ and determined by RT PCR. Statistical analysis was performed using the Kruskal-Wallis test with Dunn's multiple comparisons, Wilcoxon paired or Mann-Whitney unpaired $t$-test. Values of $\mathrm{p}<0.05(*), \mathrm{p}<0.01(* *), \mathrm{p}<0.001$ $(* * *)$ and $\mathrm{p}<0.0001(* * * *)$ were considered statistically significant.

IL-27Ralpha levels were mirrored by IL-27 levels and were able to detect elevated levels of IL-27 in T1D patients' serum (Fig. 1E). Moreover, analysis of soluble IL-27Ralpha levels in patient $(n=40)$ and healthy $(n=32)$ serum revealed that T1D patients also had increased levels of the soluble form receptor in their periphery compared to the controls (Fig. 1F). Even though, the source of sIL-27Ralpha in serum is indistinguishable, we noticed that T1D T cells expressed significantly lower levels of IL-27Ralpha on their surface in comparison to healthy donors (Suppl. Fig. 1C). Intense shedding of the IL-27Ralpha from T1D T cells might be partially responsible for the increased serum levels of sIL-27Ralpha, however the ADAM17 expression on T cells remain on the control range (data not shown).

Elevated STAT1 and 3 phosphorylation and expression of STAT1- and 3-induced genes. To uncover whether increased IL-27Ralpha expression has an impact on cell signaling, we analyzed STAT1 and STAT3 phosphorylation in mDCs of T1D patients $(n=11)$ and healthy donors $(n=11)$ using phosphoflow. We also analyzed pSTAT1 and pSTAT3 in plasmacytoid DCs (pDCs) as a control. The gating strategy is shown in Suppl. Fig. 1D. Upon rhIL-27 exposure, the STAT1 and STAT3 proteins were phosphorylated in DCs within 5 minutes and phosphorylation continued to rise with the maximum level reached in 15 minutes (Suppl. Fig. 2A). While analyzing the differences between patients and controls in the reactivity to rhIL-27 stimulation, we observed more profound STAT1 and STAT3 phosphorylation in patient mDCs compared to healthy control mDCs. Moreover, we also noticed slightly higher basal STAT3 phosphorylation in T1D mDCs (Fig. 2A). In pDCs, we did not detect any significant effect of rhIL-27 stimulation (data not shown) in comparison to controls. The effect of rhIL-27 stimulation on the PBMC compartment as detected by western blot is shown in Suppl. Fig. 2B and 3. To exclude impaired Janus kinases (JAK) activity as an underlying cause of the increased STAT3 phosphorylation, we stimulated T1D and control samples with rhIL-6 or rhIFN $\alpha$ to examine STAT3 and STAT1 phosphorylation, respectively. Both groups reacted to the cytokine exposure, however no differences between T1D and HD were detected (Suppl. Fig. 2C), suggesting that the detected increase of STAT3 activity in T1D mDCs is directly due to IL-27 stimulation and not due to a general JAK hyperactivation in T1D patients.

To confirm the effect of rhIL-27 on the signal transduction through STAT1 and STAT3 molecules, we analyzed the expression of STAT1- and STAT3-induced genes CXCL10 and SOCS3, respectively, in the PBMCs of T1D patients $(n=16)$ and healthy donors $(n=9)$ upon rhIL-27 stimulation. Both groups were able to upregulate the expression of the genes; however, in T1D patients, the increase was more significant (Fig. 2B). To exclude the possible role of poor control of glucose metabolism in T1D patients in observed impaired IL-27 signaling, we correlated glycated hemoglobin (HbA1c) levels with STAT1, 3 phosphorylation, CXCL10 and SOCS3 expression. We have not found any significant relationship between glucose metabolism and IL-27 signaling (data not shown). Moreover, we also performed similar set of experiments with rhIL-27 stimulation on T2D samples to confirm that 
A

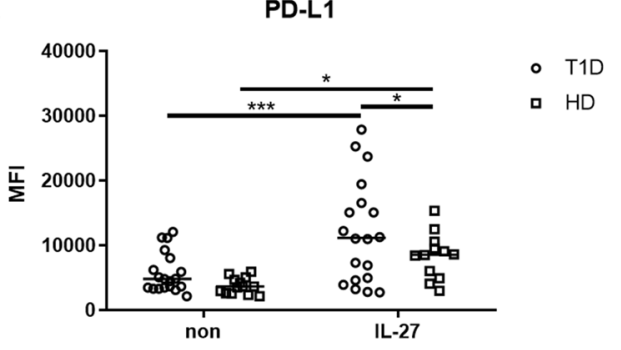

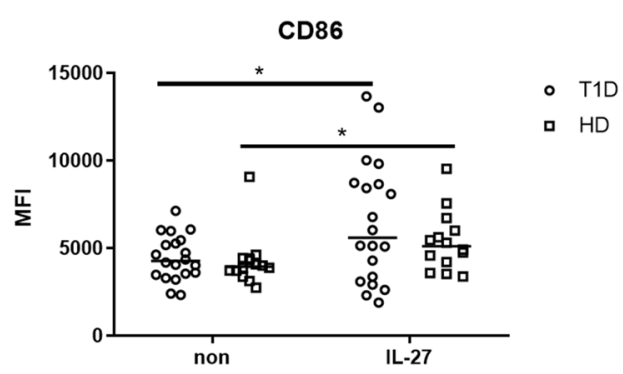

B
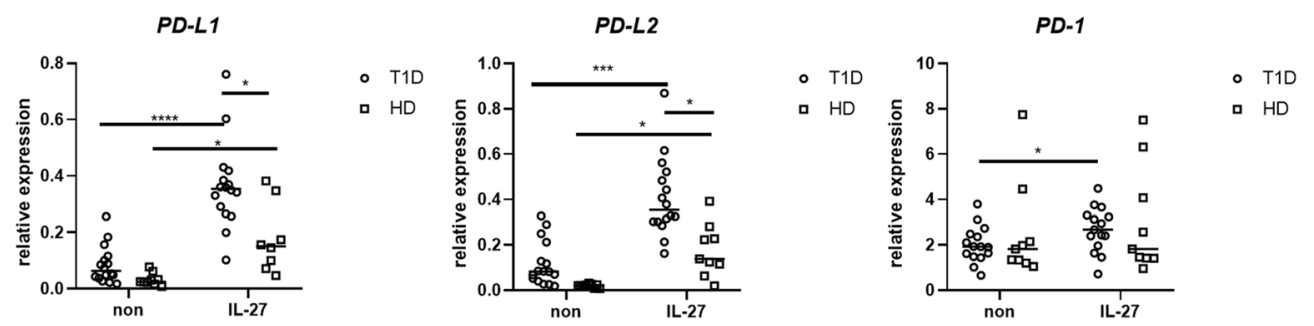

C

PD-1 expression

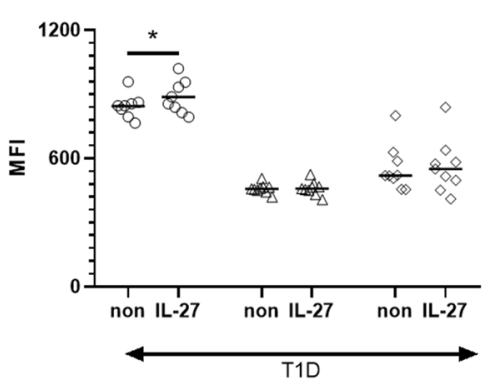

PD-1 expression

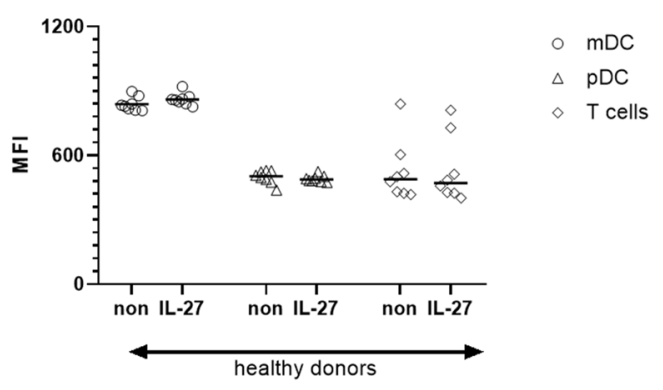

Figure 3. PD-L1 and CD86 expression on the cell surface. (A) After overnight incubation of PBMCs from T1D patients $(\mathrm{n}=20)$ and healthy donors $(\mathrm{n}=14)$ with rhIL-27 $(100 \mathrm{ng} / \mathrm{ml})$, the expression of PD-L1 and CD86 on the surface of mDCs was analyzed by flow cytometry. (B) The expression of $P D-L 1, P D-L 2$ and $P D-1$ normalized to GADPH was obtained by RT PCR in PBMCs from T1D patients $(n=16)$ and healthy donors $(\mathrm{HD}, \mathrm{n}=9)$ after stimulation with rhIL-27 (100 ng/ml) for 5 hours. (C) PD-1 expression on surface of $\mathrm{mDC}$, $\mathrm{pDC}$ and $\mathrm{T}$ cells was analyzed by flow cytometry in T1D patients $(\mathrm{n}=9)$ and $\mathrm{HD}(\mathrm{n}=8)$ after overnight incubation with $100 \mathrm{ng} / \mathrm{ml}$ rhIL-27 or left untreated. Statistical analysis was performed using the Kruskal-Wallis test with Dunn's multiple comparisons the Wilcoxon paired or Mann-Whitney unpaired $t$-test. Values of $\mathrm{p}<0.05(*), \mathrm{p}<0.01$ $(* *), \mathrm{p}<0.001(* * *)$ and $\mathrm{p}<0.0001(* * * *)$ were considered statistically significant.

impaired IL-27 reactivity of T1D mDCs is not due to poor glucose control. As we did not notice any noteworthy differences between T2D patients and healthy donors, suggesting that observed increased STAT phosphorylation in patients' $\mathrm{mDCs}$ is T1D specific (Suppl. Fig. 4B).

IL-27 induces PD-L1 expression. Using flow cytometry, we detected the expression of PD-L1 and CD86 after rhIL-27 exposure on the surface of mDCs (Fig. 3A) from T1D patients $(n=19)$ and healthy controls $(\mathrm{n}=14)$. Both T1D patients and healthy donors (HD) were able to significantly upregulate the expression of PD-L1 on the surface of mDCs in response to rhIL-27 stimulation; however the raise on T1D mDCs was more profound (Fig. 3A). There was no significant difference in PD-L1 on pDC's surface (data not shown) between healthy donors and T1D patients upon rhIL-27 exposure. Moreover, we also analyzed CD86 expression on both populations of DCs and observed that even though rhIL-27 increases CD86 expression, there were no statistically significant differences between T1D and HD (Fig. 3A). Similarly to STATs phosphorylation, we did not find any relationship between HbA1c levels and PD-L1 nor CD86 expression on mDCs. Phenotype changes of T2D patients in response to rhIL-27 stimulation were within the control range (Suppl. Fig. 4C).

Next, we analyzed the expression of programmed death-1 (PD) family members, $P D-L 1, P D-L 2$ and $P D-1$ upon rhIL-27 exposure in the PBMC compartment (Fig. 3B) by real-time PCR. PD- $L 1$ and $P D-L 2$ were significantly upregulated after rhIL-27 stimulation in both T1D patients $(n=16)$ and healthy controls $(n=9)$, but the IL-27-induced expression was significantly higher in T1D patients. Moreover, $P D-1$ expression was induced by IL-27 only in T1D patients and not in healthy donors (Fig. 3B,C). The expression of $P D-1$ negatively correlated with $\mathrm{HbAc1}$ levels in both unstimulated as well as rhIL-27 stimulated state, but no relationship was found between metabolic control and $P D-L 1$ and $P D-L 2$ expression (data not shown). 
Since PD-1 is also expressed on T cells as an activation marker, we executed additional analysis of rhIL-27 effect on PD-1 expression on various cell populations - mDC, pDC and T cells by flow cytometry and found that only T1D mDCs are able to upregulate PD-1 expression on their surface as an effect of rhIL-27 stimulation (Fig. 3C).

\section{Discussion}

IL-27 is a potent immunoregulator that has drawn interest as a target for immunotherapy ${ }^{15}$. Therefore, understanding its activity and biological role under physiological and disease conditions is essential. In this study, we show enhanced expression and signaling of IL-27Ralpha in myeloid dendritic cells (mDCs) of patients with type 1 diabetes (T1D). Increased STAT1 and STAT3 phosphorylation was accompanied by an increased expression of PD-L1 on the surface of mDCs. Interestingly, we also observed elevated expression of inhibitory receptors in T1D PBMCs in response to IL-27 stimulation, potentially suggesting a role for IL-27Ralpha signaling in the tolerogenic compensatory control of T1D.

IL-27 has been implicated in the pathologies of several autoimmune diseases, such as multiple sclerosis, systemic lupus erythematosus, rheumatoid arthritis, autoimmune gastritis and inflammatory bowel disease ${ }^{15}$. The exact mechanisms by which IL-27 contributes to the pathogenesis of these autoimmune diseases require further investigation because results from experimental models of autoimmune diseases have shown contradictory and not fully defined dual roles of IL- $27^{20}$. Both pro- and anti-inflammatory activities have been reported, and the effect of IL-27 was often context-dependent, including in the studies focused on T1D. In NOD mice, IL-27 was reported to have a pathogenic role ${ }^{16}$, but in contrast, another report showed that mice lacking IL-27 signaling displayed diabetic symptoms, such as increased blood sugar levels, islet infiltration, proinflammatory cytokine production, and administration of IL-27 reversed these symptoms ${ }^{17}$, suggesting that targeting the IL-27 pathway could be a potential therapeutic strategy. One study performed in humans reported increased IL-27 levels in the peripheral blood of newly diagnosed T1D patients ${ }^{3}$, which is in agreement with our observation of elevated IL-27 level in our cohort of long-term treated T1D patients. Interestingly, we have previously identified IL-27 signaling as one of the pathways distinguishing two siblings who had already developed T1D out of four HLA identical quadruplets ${ }^{21}$. Elevated levels of IL-27 mRNA were also found in patients with active Crohn's disease and an enhanced percentage of IL-27 immunoreactive cells in the gut mucosa in patients with active ulcerative colitis. However, the authors of the study suggested that enhanced IL-27 expression had an anti-inflammatory role in inflammatory bowel diseases due to the compensatory mechanism of the inflammatory process in the intestine ${ }^{22}$.

Increased IL-27 serum level was mirrored by elevated amount of the natural antagonist of IL-27, a soluble form of IL-27Ralpha, in long-term treated T1D patients. Soluble IL-27Ralpha (sIL-27Ralpha) has the ability to bind recombinant human IL-27, inhibit its binding to surface receptors and consequently block its STAT-mediated signaling ${ }^{11}$. Elevated levels of sIL-27Ralpha were also found in the sera of patients with Crohn's disease, suggesting that this molecule may play a role in immunopathologies beyond $\mathrm{T} 1 \mathrm{D}^{11}$.

Through gene microarrays, we detected increased expression of IL-27Ralpha in purified mDCs from T1D patients. IL-27Ralpha is constitutively expressed by numerous immune and nonimmune cell types ${ }^{11}$, and upon IL-27 stimulation, it activates the JAK/STAT and MAPK signaling pathways ${ }^{10}$. In this study, we observed elevated STAT1 and STAT3 phosphorylation after IL-27 exposure in T1D mDCs. Elevated STAT3 phosphorylation was also detected in unstimulated cells. STAT3 signaling has been shown to induce a tolerogenic phenotype in DCs, and conversely, STAT3 deficiency leads to the development of inflammation ${ }^{23}$. This might again suggest a tolerogenic role of IL-27 as a STAT3 activator in DCs. Moreover, IL-27 is a known inducer of PD-L1 expression in various cell types, acting through STAT1 activation ${ }^{24-26}$, further implying an anti-inflammatory role for IL-27. Indeed, in vitro stimulation of T1D cells with IL-27 led not only to increased expression of $P D-L 1$ but also of $P D-L 2$, and the expression was even further increased in T1D patients. Surprisingly, $P D-1$ expression was also induced by IL-27; however, this effect was exclusive to $\mathrm{mDCs}$ of T1D patients. These inhibitory receptors are essential for regulating $\mathrm{T}$ cell activation and promoting immune tolerance. The PD-1/PD-L1 pathway regulates the induction and maintenance of peripheral immune tolerance and protects tissues against autoimmune attacks ${ }^{27}$. Mounting evidence has shown that impaired function of PD-1/PD-L1 signaling plays an important role in several autoimmune diseases, including T1D $28-31$.

Previous studies have associated IL-27 effect on regulatory T lymphocytes (Tregs) with both pro- and anti-inflammatory roles. The IL-27 signaling might contribute to their suppressive activities via STAT1-dependent manner ${ }^{32,33}$ or by attenuating the Th17-mediated inflammation as shown in an autoimmune model of experimental autoimmune encephalomyelitis ${ }^{12}$. On the other hand, Bin Dhuban et al. showed that IL-27 impairs the suppressive capacity of human Treg via the gp 130 receptor subunit ${ }^{34}$. Even though we did not focus on Treg reactivity to IL-27 in our experimental setup, it is therefore plausible that Treg contribution to T1D pathology might be influenced by enhanced IL-27 signaling in T1D patients, however the exact spectrum of effects is still elusive and needs further investigation.

Thus, in this study, we show evidence of enhanced in vitro IL-27 signaling in T1D patient cells. Elevated IL-27Ralpha expression on the surface of mDCs, increased STAT3 phosphorylation and preeminent expression of inhibitory receptors after IL-27 stimulation suggest compensatory mechanisms due to ongoing inflammation and immunomodulatory role of IL-27 in T1D pathogenesis; however, the precise function of IL-27 in vivo needs further investigation.

\section{Patients and Methods}

Patients. A cohort of 51 pediatric patients with T1D (62.3\% female) and 44 healthy donors (52.4\% female) was included in this study. The median age of patients with T1D was $15.4 \pm 2.3$ years (range: 9.6-18.6 years), and the median age of the healthy donors was $18.5 \pm 3.0$ years (range: $12.2-21.6$ years). All patients with T1D had been treated with insulin since disease onset. The median T1D duration was 5.9 \pm 3.3 years (range: 1.2-15.3 
years). The median of the last glycated hemoglobin (HbA1c) was $64 \pm 14 \mathrm{mmol} / \mathrm{mol}$ (range: 39-94 mmol/mol). At the time of blood sampling, patients were metabolically stable; none of them had signs of active infection, neoplasia or other comorbidities except well-controlled celiac disease or autoimmune thyroiditis (5\% of recruited patients with T1D). The healthy donors had a negative personal history of autoimmune diseases. Moreover, we included a cohort of T2D patients to the study. The median age of the patients was $54.8 \pm 11.3$ years (range: 42.6-75.9 years) with median duration of the disease $4.9 \pm 5.8$ years (range: $1.57-18.7$ ). The median of glycated hemoglobin was $46.5 \pm 8 \mathrm{mmol} / \mathrm{mol}$. Not all patients were involved in all experiments due to the limited amount of blood available per sample.

Written informed consent was obtained from all the patients or the patients' parents/guardians in accordance with the Declaration of Helsinki, and the study was approved by the Ethics Committee of University Hospital Motol.

Cell isolation and culture. Peripheral blood was collected from patients and healthy volunteers into EDTA-coated tubes. Peripheral blood mononuclear cells (PBMCs) were isolated using Ficoll-Paque (GE Healthcare Biosciences, Uppsala, Sweden). The obtained cells were resuspended in RPMI 1640 medium with a sodium bicarbonate buffer system supplemented with $2 \%$ autologous serum, $1 \%$ penicillin and streptomycin and 1\% Glutamax (ThermoFisher Scientific, Waltham, USA).

Plasmacytoid and myeloid DC purification. PBMCs isolated from 20 T1D patients and 10 healthy controls were stained with FITC-conjugated antibodies against lineage-specific markers (CD3 clone MEM-57, CD19 clone LT19, CD20 clone LT20, CD16 clone LNK16, and CD56 clone MEM-188), anti-CD14-PEDy594 (clone MEM-15), anti-CD11c-APC (clone BU15) (Exbio, Prague, Czech Republic), anti-CD123-PE (clone 6H6) (ThermoFisher Scientific), and anti-HLA-DR-PC7 (clone L243) (BD Biosciences, Franklin Lakes, USA), washed, filtrated through $0.2 \mu \mathrm{m}$ pore size and sorted under low pressure to increase viability and prevent activation on a FACSAria II (BD Biosciences). Myeloid DCs were gated as Lin-HLA-DR + CD14-CD11c+ cells, and plasmacytoid DCs were defined as Lin-HLA-DR + CD14-CD123+ cells. Sorted cells were recollected in tubes containing complete RPMI.

Gene arrays. RNA was isolated from sorted mDCs and pDCs using the RNeasy Micro Kit following the manufacturer's instructions (Qiagen, MD, USA). RNA concentration was measured with a spectrophotometer (Nanodrop), and RNA integrity was assessed by an Agilent 2100 bioanalyzer (Agilent, Santa Clara, USA). Total RNA was amplified (aRNA) using an Amino AllyI MessageAmp II aRNA Amplification Kit (Applied Biosystems, Foster City, USA). The RNA was then stained with Alexa 555 (Molecular Probes, Eugene, USA) and hybridized to a SurePrint G3 Human Gene Expression $8 \times 60 \mathrm{~K}$ v2 Microarray (Agilent) according to the manufacturer's instructions. The microarray was then scanned using InnoScan 900 (Innopsys, Carbonne, France). Microarray data processing and statistical analysis of differential gene expression were performed using the lima package in the R statistical program. Two-color microarray data analysis was performed according to the manufacturer's recommendations. For each chip, raw intensity data were corrected for background, normalized by intra-array loess normalization and subjected to intra-array quantile normalization.

IL-27 and soluble IL-27Ralpha serum levels. The concentration of sIL-27Ralpha in T1D sera was determined by ELISA (Sigma Aldrich, St. Luis, USA) following the manufacturer's instructions. IL-27 was determined by Luminex (ThermoFisher).

PD-L1 expression. PBMCs were cultured in complete media with or without rhIL-27 (100 ng/ml) overnight at $37^{\circ} \mathrm{C}$. Then, the cells were washed and stained with FITC-conjugated antibodies against lineage-specific markers (CD3 clone MEM-57, CD19 clone LT19, CD20 clone LT20, CD16 clone LNK16, and CD56 clone MEM188), anti-CD14-PEDy594 (clone MEM-15), anti-CD11c-PB (clone BU15) (Exbio), anti-CD123-PerCP-Cy5.5 (clone 7G3) (BD Biosciences), anti-HLA-DR-Alexa700 (clone L243), anti-PD-L1-PC7 (clone 29E.2A3), and anti-CD86-Alexa647 (clone IT2.2) (BioLegend, San Diego, USA). The samples were acquired on a FACSAria II (BD Biosciences), and data analysis was performed using FlowJo software (TreeStar). Next, the expression of $P D-L 1$ in PBMCs was analyzed by RT-PCR after $100 \mathrm{ng} / \mathrm{ml}$ IL-27 stimulation for 5 hours.

Surface marker expression. PBMCs were cultured in complete media with or without rhIL-27 (100 ng/ $\mathrm{ml}$ ) overnight at $37^{\circ} \mathrm{C}$ and following antibodies were used: lineage-specific markers conjugated with FITC (CD3 clone MEM-57, CD19 clone LT19, CD20 clone LT20, CD16 clone LNK16, and CD56 clone MEM-188), anti-CD14-PEDy594 (clone MEM-15), anti-CD3-Alexa700 (clone MEM-15), anti-CD11c-APC (clone BU15) anti-CD86-PE (clone BU63) (Exbio), anti-CD123-PC7 (clone 6H6), anti-PD1-BV421 (clone EH12.2H7), anti-PDL1-BV510 (clone 29E.2A3), anti-gp130-PE (clone 2E1B02) (BioLegend), anti-HLA-DR-PerCP (BD Biosciences), anti-IL27Ralpha-PE (clone 191106) and anti-ADAM17-PE (clone 111633) (R\&D Systems, Minneapolis, USA), respectively. The samples were acquired on a FACSFortessa (BD Biosciences), and data analysis was performed using FlowJo software (TreeStar).

Phosphoflow. Whole blood was stimulated with $100 \mathrm{ng} / \mathrm{ml}$ rhIL-27 (Peprotech, New York, USA) for 15 minutes, $10 \mathrm{ng} / \mathrm{ml} \mathrm{rhIL}-6,500 \mathrm{ng} / \mathrm{ml} \mathrm{rhIFN \alpha}$ (both from R\&D Systems) when indicated or left untreated at $37^{\circ} \mathrm{C}$. The optimal stimulation time was estimated after analyzing the effects of 5, 15 and 30 minutes of rhIL-27 stimulation on 8 healthy controls (Suppl. Fig. 2A). Intracellular signaling was prevented by using $4 \%$ paraformaldehyde without methanol (Sigma Aldrich) for 10 minutes at room temperature. Erythrocytes were lysed using $0,1 \%$ Triton-X for 20 minutes (Sigma Aldrich) at $37^{\circ} \mathrm{C}$, leukocytes were permeabilized with ice-cold $80 \%$ methanol for 30 minutes and stained with a FITC-conjugated lineage antibody cocktail (CD3 clone MEM-57, CD19 clone 
LT19, CD20 clone LT20, CD16 clone LNK16, and CD56 clone MEM-188), anti-CD14-PEDy594 (clone MEM15) (Exbio), anti-HLA-DR-Alexa700 (clone L243), anti-CD123-PC7 (clone 6H6) (BioLegend), anti-CD11c-APC (clone BU15) (Exbio), anti-phosphoSTAT1-BV421 (Tyr701) (clone 4a) and anti-phosphoSTAT3-PE (Tyr705) (clone 4/5-STAT3) (both from BD Bioscience). The samples were acquired on FACSAria II (BD Biosciences), and data analysis was performed using FlowJo (TreeStar).

Western blot. PBMCs were stimulated for 15, 30 and 45 minutes with rhIL-27 (100 ng/ml) or left untreated. The cells were then washed and lysed with RIPA lysis buffer and PMSF (Cell Signaling, Danvers, USA), placed on ice, sonicated and then centrifuged at $14000 \mathrm{~g}$ to remove cell debris. For western blot analysis, samples were resuspended in Laemmli buffer (Sigma Aldrich) at a 1:1 ratio and boiled for $5 \mathrm{~min}$. Proteins were separated by SDS-PAGE and transferred to PVDF membranes. After blocking with 5\% BSA in TBST (TBS and 0.1\% Tween, both from Bio-Rad, Hercules, USA), the membranes were incubated with the following primary antibodies: anti-STAT1 (clone EPYR2154) (Abcam), anti-phosphoSTAT1 (Tyr701) (clone M135), anti-STAT3 (clone D3Z2G), anti-phosphoSTAT3 (Tyr705) (clone D3A7) and anti- $\beta$-actin (clone D6A8) (all from Cell Signaling) overnight, followed by incubation with peroxidase-conjugated anti-rabbit or anti-mouse secondary antibodies for 2 hours. Membranes were developed using SuperSignal West Femto (ThermoFisher Scientific). Densitometry was performed with ImageJ software (National Institutes of Health, USA). Protein band area values were used for semiquantification. Graphs represent the ratio of stimulated/unstimulated cells of the band area calculated from the band area of phosphorylated forms/band area of unphosphorylated forms.

RT-PCR. PBMCs were stimulated for 5 hours with rhIL-27 $(100 \mathrm{ng} / \mathrm{ml})$ or left untreated. RNA isolation, reverse transcription and RT-PCR were performed according to a previously published protocol ${ }^{35}$. Total RNA was isolated using the RNeasy Mini Kit following the manufacturer's instructions (Qiagen, MD, USA), and complementary DNA (cDNA) was synthetized using M-MLV Reverse Transcriptase (ThermoFisher Scientific). RT-PCR was performed in duplicate using the obtained cDNA and Platinum Taq polymerase (ThermoFisher Scientific), $200 \mathrm{nM}$ dNTP (Promega, Southampton, UK), $50 \mathrm{mM} \mathrm{MgCl}_{2}$ (ThermoFisher Scientific) and TaqMan primer/probe sets (ThermoFisher Scientific). Samples were matched to a standard curve generated by amplifying serially diluted products using the same PCR and normalized to GAPDH (forward primer GAAGGTGAAGGTCGGAGTC; reverse primer GAAGATGGTGATGGGATTTC; FAM/TAMRA CAAGCTTCCCGTTCTCAGCC) (TIB MOLBIOL, Berlin, Germany) to obtain the relative expression value. Real-time assays were run on an FX96 Cycler (Bio-Rad). The following primer/probe sets were used: IL-27Ralpha (Hs00956025_m1), CXCL10 (Hs00171042_ m1), SOCS3 (Hs02330328_s1), PD-L1 (Hs01125301_m1), PD-L2 (Hs00228836_m1), PD-1 (Hs00169472_m1), all from ThermoFisher Scientific.

Statistics. The results were obtained from at least six independent experiments. Not all patients were involved in all experiments due to the limited amount of blood available per sample. Statistical analysis was performed using non-parametric one-way analysis of variance (ANOVA) with multiple comparisons Dunn's post-test where applicable. A two-tailed paired Wilcoxon or unpaired Mann-Whitney $t$-test was also applied for data analysis using GraphPad Prism 8. Values of $\mathrm{p}<0.05(*), \mathrm{p}<0.01(* *) \mathrm{p}<0.001(* * *)$ and $\mathrm{p}<0.0001$ (****) were considered statistically significant.

Received: 12 August 2019; Accepted: 2 January 2020;

Published online: 16 January 2020

\section{References}

1. He, J.-S. et al. Role of immune dysfunction in pathogenesis of type 1 diabetes mellitus in children. Asian Pac. J. Trop. Med. 7, 823-826 (2014).

2. CAVALLO, M. G. et al. Cytokines in sera from insulin-dependent diabetic patients at diagnosis. Clin. Exp. Immunol. 86, 256-259 (2008).

3. Alnek, K. et al. Increased Blood Levels of Growth Factors, Proinflammatory Cytokines, and Th17 Cytokines in Patients with Newly Diagnosed Type 1 Diabetes. PLoS One 10, e0142976 (2015).

4. Devergne, O. et al. A novel interleukin-12 p40-related protein induced by latent Epstein-Barr virus infection in B lymphocytes. J. Virol. 70, 1143-53 (1996).

5. Pflanz, S. et al. IL-27, a Heterodimeric Cytokine Composed of EBI3 and p28 Protein, Induces Proliferation of Naive CD4+ T Cells. Immunity 16, 779-790 (2002).

6. Takeda, A. et al. Cutting edge: role of IL-27/WSX-1 signaling for induction of T-bet through activation of STAT1 during initial Th1 commitment. J. Immunol. 170, 4886-90 (2003).

7. Awasthi, A. et al. A dominant function for interleukin 27 in generating interleukin 10-producing anti-inflammatory T cells. Nat. Immunol. 8, 1380-1389 (2007).

8. Murugaiyan, G. et al. IL-27 is a key regulator of IL-10 and IL-17 production by human CD4+ T cells. J. Immunol. 183, 2435-43 (2009).

9. Stumhofer, J. S. et al. Interleukin 27 negatively regulates the development of interleukin 17-producing T helper cells during chronic inflammation of the central nervous system. Nat. Immunol. 7, 937-945 (2006).

10. Pflanz, S. et al. WSX-1 and glycoprotein 130 constitute a signal-transducing receptor for IL-27. J. Immunol. 172, 2225-31 (2004).

11. Dietrich, C., Candon, S., Ruemmele, F. M. \& Devergne, O. A Soluble Form of IL-27R Is a Natural IL-27 Antagonist. J. Immunol. 192, 5382-5389 (2014).

12. Fitzgerald, D. C. et al. Suppressive effect of IL-27 on encephalitogenic Th17 cells and the effector phase of experimental autoimmune encephalomyelitis. J. Immunol. 179, 3268-75 (2007).

13. Fitzgerald, D. C. et al. Suppression of autoimmune inflammation of the central nervous system by interleukin 10 secreted by interleukin 27-stimulated T cells. Nat. Immunol. 8, 1372-1379 (2007).

14. Mascanfroni, I. D. et al. IL-27 acts on DCs to suppress the T cell response and autoimmunity by inducing expression of the immunoregulatory molecule CD39. Nat. Immunol. 14, 1054-1063 (2013).

15. Meka, R. R., Venkatesha, S. H., Dudics, S., Acharya, B. \& Moudgil, K. D. IL-27-induced modulation of autoimmunity and its therapeutic potential. Autoimmun. Rev. 14, 1131-1141 (2015). 
16. Wang, R. et al. The pathogenic role of interleukin-27 in autoimmune diabetes. Cell. Mol. Life Sci. 65, 3851-3860 (2008).

17. Fujimoto, H. et al. IL-27 Inhibits Hyperglycemia and Pancreatic Islet Inflammation Induced by Streptozotocin in Mice. Am. J. Pathol. 179, 2327-2336 (2011).

18. Reddy, M. V. P. L. et al. Association between type 1 diabetes and GWAS SNPs in the southeast US Caucasian population. Genes Immun. 12, 208-12 (2011).

19. Santos, A. S. et al. Lack of association between IL27 gene variants and type 1 diabetes susceptibility. Cytokine 61, 349-352 (2013).

20. Ejtehadifar, M. et al. IL-27: Friend or Foe in the Autoimmune Diseases. Curr. Immunol. Rev. 13 (2018).

21. Stechova, K. et al. Case report: Type 1 diabetes in monozygotic quadruplets. Eur. J. Hum. Genet. 20 (2012).

22. Furuzawa Carballeda, J., Fonseca Camarillo, G. \& Yamamoto-Furusho, J. K. Interleukin 27 is up-regulated in patients with active inflammatory bowel disease. Immunol. Res. 64, 901-907 (2016).

23. Melillo, J. A. et al. Dendritic Cell (DC)-Specific Targeting Reveals Stat3 as a Negative Regulator of DC Function. J. Immunol. 184, 2638-2645 (2010).

24. DeLong, J. H. et al. IL-27 and TCR Stimulation Promote T Cell Expression of Multiple Inhibitory Receptors. ImmunoHorizons 3, $13-25$ (2019)

25. Hirahara, K. et al. Interleukin-27 Priming of T Cells Controls IL-17 Production In trans via Induction of the Ligand PD-L1. Immunity 36, 1017-1030 (2012).

26. Carbotti, G. et al. IL-27 induces the expression of IDO and PD-L1 in human cancer cells. Oncotarget 6, 43267-80 (2015).

27. Chen, L. \& Flies, D. B. Molecular mechanisms of T cell co-stimulation and co-inhibition. Nat. Rev. Immunol. 13, 227-242 (2013).

28. Guleria, I. et al. Mechanisms of PDL1-mediated regulation of autoimmune diabetes. Clin. Immunol. 125, 16-25 (2007).

29. Wang, J. et al. Establishment of NOD-Pdcd1-/- mice as an efficient animal model of type I diabetes. Proc. Natl. Acad. Sci. 102, 11823-11828 (2005).

30. Yadav, D., Hill, N., Yagita, H., Azuma, M. \& Sarvetnick, N. Altered availability of PD-1/PD ligands is associated with the failure to control autoimmunity in NOD mice. Cell. Immunol. 258, 161-171 (2009).

31. Kadri, N. et al. CD4 ${ }^{+}$Type II NKT Cells Mediate ICOS and Programmed Death-1-Dependent Regulation of Type 1 Diabetes. J. Immunol. 188, 3138-3149(2012).

32. Nguyen, Q. T. et al. IL-27 targets Foxp3+ Tregs to mediate antiinflammatory functions during experimental allergic airway inflammation. JCI Insight 4 (2019).

33. Park, Y.-J. et al. IL-27 confers a protumorigenic activity of regulatory T cells via CD39. Proc. Natl. Acad. Sci. USA 116, 3106-3111 (2019).

34. Bin Dhuban, K., Bartolucci, S., d'Hennezel, E. \& Piccirillo, C. A. Signaling Through gp130 Compromises Suppressive Function in Human FOXP3+ Regulatory T Cells. Front. Immunol. 10, 1532 (2019).

35. Zentsova, I. et al. Monocytes contribute to DNA sensing through the TBK1 signaling pathway in type 1 diabetes patients. J. Autoimmun. https://doi.org/10.1016/j.jaut.2019.06.005 (2019).

\section{Acknowledgements}

The study was supported by the Czech Ministry of Health AZV 16-32838A, GAUK 460218 issued by Charles University in Prague, Czech Republic. We thank all the patients and healthy volunteers for the blood samples used in this study. We extend special thanks to Adam Klocperk for his help and valuable comments. We confirm that this manuscript has not been published elsewhere and is not under consideration by another journal.

\section{Author contributions}

Z.P. designed the study and experiments, performed experiments, analyzed the data, interpreted the results and wrote the manuscript. P.V. and I.Z. performed the experiments. J.K. performed the cell sorting. L.S. and I.R. analyzed the gene arrays. K.S. provided T2D patient biological material and information. Z.S. provided T1D patient information and biological material and reviewed the manuscript. A.S. designed the study and reviewed and edited the manuscript.

\section{Competing interests}

The authors declare no competing interests.

\section{Additional information}

Supplementary information is available for this paper at https://doi.org/10.1038/s41598-020-57507-8.

Correspondence and requests for materials should be addressed to Z.P.

Reprints and permissions information is available at www.nature.com/reprints.

Publisher's note Springer Nature remains neutral with regard to jurisdictional claims in published maps and institutional affiliations.

(c) (i) Open Access This article is licensed under a Creative Commons Attribution 4.0 International License, which permits use, sharing, adaptation, distribution and reproduction in any medium or format, as long as you give appropriate credit to the original author(s) and the source, provide a link to the Creative Commons license, and indicate if changes were made. The images or other third party material in this article are included in the article's Creative Commons license, unless indicated otherwise in a credit line to the material. If material is not included in the article's Creative Commons license and your intended use is not permitted by statutory regulation or exceeds the permitted use, you will need to obtain permission directly from the copyright holder. To view a copy of this license, visit http://creativecommons.org/licenses/by/4.0/.

(C) The Author(s) 2020 